\title{
Some Properties of Solutions for Some $q$-Difference Equations Containing Painlevé Equation
}

\author{
Peng Jun Zhao ${ }^{1}$ and Hong Yan $\mathrm{Xu} \mathbb{i D}^{2}$ \\ ${ }^{1}$ School of Mathematics and Computer Applications, Shangluo University, Shangluo, Shaanxi 726000, China \\ ${ }^{2}$ Department of Informatics and Engineering, Jingdezhen Ceramic Institute, Jingdezhen, Jiangxi 333403, China \\ Correspondence should be addressed to Hong Yan Xu; xhyhhh@126.com
}

Received 4 January 2018; Revised 7 March 2018; Accepted 28 March 2018; Published 7 May 2018

Academic Editor: Richard I. Avery

Copyright (c) 2018 Peng Jun Zhao and Hong Yan Xu. This is an open access article distributed under the Creative Commons Attribution License, which permits unrestricted use, distribution, and reproduction in any medium, provided the original work is properly cited.

\begin{abstract}
The existence and growth of meromorphic solutions $f(z)$ for some $q$-difference equations are studied, and some estimates for the exponent of convergence of poles of $\Delta_{q} f, \Delta_{q}^{2} f, \Delta_{q} f / f$, and $\Delta_{q}^{2} f / f$ are also obtained. Our theorems are improvements and extensions of the previous results.
\end{abstract}

\section{Introduction and Main Results}

In 1900, Painlevé [1] first studied the differential equations, which were called differential Painlevé equations later. Moreover, at the beginning of last century, differential Painlevé equations had been an important research subject in the field of the mathematics and physics. They occur in many physical situations, such as plasma physics, statistical mechanics, and nonlinear waves.

In the 1990s, the discrete Painlevé equations had become important and interest research problems (see [2, 3]). For example, let $a, b$, and $c$ be constants and $n \in \mathbb{N}_{+}$, it is usual that

$$
\begin{aligned}
& y_{n+1}+y_{n-1}=\frac{a n+b}{y_{n}}+c, \\
& y_{n+1}+y_{n-1}=\frac{a n+b}{y_{n}}+\frac{c}{y_{n}^{2}}
\end{aligned}
$$

are called the special discretization of discrete $P_{I}$, and

$$
y_{n+1}+y_{n-1}=\frac{(a n+b) y_{n}+c}{1-y_{n}^{2}}
$$

is called the special discretization of the discrete $P_{I I}$.
Of late, with the development of Nevanlinna theory, Chiang and Feng [4] and Halburd and Korhonen [5] established independently those results about the difference analog of the lemma on the logarithmic derivative, and there has been an increasing interest in studying complex difference equations. And there were a number of papers (see $[4,6$, 7]) concerning complex difference equations and difference analogs of Nevanlinna theory, by applying the results of Chiang and Feng [4] and Halburd and Korhonen [5]. For example, Halburd and Korhonen [5, 8, 9] used Nevanlinna theory to analyze the following equation:

$$
w(z+1)+w(z-1)=R(z, w),
$$

where $R(z, w)$ is rational in $w$ and meromorphic in $z$, and we single out the difference of Painleve $I$ and $I I$ equations such as

$$
\begin{aligned}
& w(z+1)+w(z-1)=\frac{a z+b}{w(z)}+c, \\
& w(z+1)+w(z-1)=\frac{a z+b}{w(z)}+\frac{c}{w(z)^{2}}, \\
& w(z+1)+w(z-1)=\frac{(a z+b) w(z)+c}{1-w(z)^{2}} .
\end{aligned}
$$

In recent, Laine and Yang, Zhang and Korhonen, and Zheng and Chen further investigated the value distribution of 
$q$-difference operator of meromorphic functions, by utilizing the analog of Logarithmic Derivative Lemma on $q$-difference operators given by Barnett et al. [10]. Moreover, during the last decades, considerable attention has been paid to $q$-difference operators, $q$-difference equations, by replacing the $q$-difference $f(q z), q \in \mathbb{C} \backslash\{0,1\}$ with $f(z+c)$ of a meromorphic function in some complex difference equations and complex difference operators (see [11-26]).

Throughout this paper, a term "meromorphic" will always mean meromorphic in the complex plane $\mathbb{C}$. Hereinafter, we will use some basic results and the standard notations of Nevanlinna theory (see [27-29]). For a meromorphic function $f$, we use $\sigma(f), \lambda(f)$, and $\lambda(1 / f)$ to denote the order, the exponent of convergence of zeros, and the exponent of convergence of poles of $f(z)$, respectively, and let $\tau(f)$ be the exponent of convergence of fixed points of $f(z)$, which is defined by

$$
\tau(f)=\limsup _{r \rightarrow+\infty} \frac{\log N(r, 1 /(f(z)-z))}{\log r} .
$$

Besides, we use $S(r, f)$ to denote any quantity satisfying $S(r, f)=o(T(r, f))$ for all $r$ outside a possible exceptional set $E$ of finite logarithmic measure

$$
\lim _{r \rightarrow \infty} \int_{[1, r) \cap E} \frac{d t}{t}<\infty,
$$

and a meromorphic function $a(z)$ is called a small function with respect to $f$ if $T(r, a(z))=S(r, f)$, and we use $\delta(f)$ to denote the field of small functions relative to $f(z)$.

In 2010, Chen and Shon [30] considered the difference Painlevé I equation (4) and obtained the following theorem.

Theorem 1 (see [30, Theorem 4]). Let $a, b$, and c be constants, where $a, b$ are not both equal to zero. Then

(i) if $a \neq 0$, then (4) has no rational solution;

(ii) if $a=0$, and $b \neq 0$, then (4) has a nonzero constant solution $w(z)=A$, where $A$ satisfies $2 A^{2}-c A-b=0$.

The other rational solution $w(z)$ satisfies $w(z)=$ $P(z) / Q(z)+A$, where $P(z)$ and $Q(z)$ are relatively prime polynomials and satisfy $\operatorname{deg} P<\operatorname{deg} Q$.

In 2015, the properties of solutions of a certain type of difference equation were further investigated by $\mathrm{Li}$ and Huang [31], and some results were obtained as follows.

Theorem 2 (see [31, Theorem 3.1]). Suppose that equation

$$
w(z+1)+w(z-1)=\frac{A(z)}{w(z)}+C(z)
$$

where $A(z), C(z) \in \mathcal{S}(w)$, admits a finite-order transcendental meromorphic solution $w(z)$. Then

(i) $\lambda(w)=\lambda(1 / w)=\sigma(w)$;

(ii) $w(z)$ has no Borel exceptional value;

(iii) if $A(z)$ \# $2 z^{2}-z C(z)$, then the exponent of convergence of fixed points of $w(z)$ satisfies $\tau(w)=\sigma(w)$.
In the same year, Qi and Yang [32] discussed the following equation:

$$
w(q z)+w\left(\frac{z}{q}\right)=\frac{a z+b}{w(z)}+c,
$$

which can be seen as $q$-difference analogs of (4), and obtained some properties of the zeros of $w(z)-A$, where $w(z)$ is a solution of (10) and $A \in \mathbb{C} \cup\{\infty\}$.

Inspired by the idea of Li and Huang [31] and Qi and Yang [32], our main purpose is further to investigate some properties of meromorphic solutions for some $q$-difference equations which are different from (10) to a certain extent, and the following theorems are obtained.

Theorem 3. Let $q \in \mathbb{C}-\{0,1\}$, and $A(z)(\not \equiv 0), C(z) \in \mathcal{S}(f)$. If

$$
f(q z)+f\left(\frac{z}{q}\right)=\frac{A(z)}{f(z)}+C(z)
$$

admits a zero-order transcendental meromorphic solution $f(z)$, then

(i) $f(z)$ has infinitely many poles and zeros, $\Delta_{q} f(z)$ also has infinitely many poles, and

$$
\lambda(f)=\lambda\left(\frac{1}{f}\right)=\lambda\left(\frac{1}{\Delta_{q} f}\right),
$$

and further, if $C(z) \neq 0$, then each of $\Delta_{q}^{2} f, \Delta_{q} f / f, \Delta_{q}^{2} f / f$ has infinitely many poles, and

$$
\lambda\left(\frac{1}{\Delta_{q}^{2} f}\right)=\lambda\left(\frac{1}{\Delta_{q} f / f}\right)=\lambda\left(\frac{1}{\Delta_{q}^{2} f / f}\right),
$$

(ii) if $A(z) \not \equiv(q+1 / q) z^{2}-z C(z)$, then $f$ has infinitely many fixed points and the exponent of convergence of fixed points of $f$ satisfies $\tau(f)=\sigma(f)$.

Theorem 4. Let $q \in \mathbb{C}-\{0,1\}$, and $A(z), C(z)(\not \equiv 0) \in$ $\mathcal{S}(f)$, and assume that $f(z)$ is a zero-order transcendental meromorphic solution of equation

$$
f(q z)+f\left(\frac{z}{q}\right)=\frac{A(z) f(z)+C(z)}{1-f(z)^{2}} .
$$

Then

(i) $f$ has infinitely many poles and zeros, $\Delta_{q} f$ also has infinitely many poles, and

$$
\lambda(f)=\lambda\left(\frac{1}{f}\right)=\lambda\left(\frac{1}{\Delta_{q} f}\right),
$$

and further, if $A(z) \not \equiv 0$, then each of $\Delta_{q}^{2} f, \Delta_{q} f / f, \Delta_{q}^{2} f / f$ has infinitely many poles, and

$$
\lambda\left(\frac{1}{\Delta_{q}^{2} f}\right)=\lambda\left(\frac{1}{\Delta_{q} f / f}\right)=\lambda\left(\frac{1}{\Delta_{q}^{2} f / f}\right),
$$

(ii) if $(q+1 / q)\left(z^{3}-z\right)+z A(z) \not \equiv-C(z)$, then $f$ has infinitely many fixed points and the exponent of convergence of fixed points of $f$ satisfies $\tau(f)=\sigma(f)$. 
Theorem 5. Let $q \in \mathbb{C}-\{0,1\}$, and $A(z)(\not \equiv 0), C(z) \in \mathcal{S}(f)$ be identically vanishing simultaneously. And $f(z)$ is a zeroorder transcendental meromorphic solution of equation

$$
f(q z)+f\left(\frac{z}{q}\right)=\frac{A(z)}{f(z)}+\frac{C(z)}{f(z)^{2}} .
$$

Then

(i) $f(z)$ has infinitely many poles and zeros, $\Delta_{q} f(z)$ also has infinitely many poles, and

$$
\lambda(f)=\lambda\left(\frac{1}{f}\right)=\lambda\left(\frac{1}{\Delta_{q} f}\right),
$$

and further, if $C(z) \neq \equiv$, then each of $\Delta_{q}^{2} f, \Delta_{q} f / f, \Delta_{q}^{2} f / f$ has infinitely many poles, and

$$
\lambda\left(\frac{1}{\Delta_{q}^{2} f}\right)=\lambda\left(\frac{1}{\Delta_{q} f / f}\right)=\lambda\left(\frac{1}{\Delta_{q}^{2} f / f}\right),
$$

(ii) if $(q+1 / q) z^{3}-z A(z) \not \equiv C(z)$, then $f$ has infinitely many fixed points and the exponent of convergence of fixed points of $f$ satisfies $\tau(f)=\sigma(f)$.

\section{Some Lemmas}

Let the logarithmic density of a set $F$ be defined by

$$
\limsup _{r \rightarrow \infty} \frac{1}{\log r} \int_{[1, r] \cap F} \frac{1}{t} d t .
$$

Definition 6 (see [10]). For $q \in \mathbb{C} \backslash\{0,1\}$, if a polynomial in $f(z)$ includes finitely many of its $q$-shifts $f(q z), \ldots, f\left(q^{n} z\right)$ with meromorphic coefficients in the sense that their Nevanlinna characteristic functions are $o(T(r, f))$ on a set $F$ of logarithmic density 1 , then it can be called a $q$-difference polynomial of $f$.

Lemma 7 (see [13, Theorem 2.5]). Let $f$ be a transcendental meromorphic solution of order zero of a q-difference equation of the form

$$
U_{q}(z, f) P_{q}(z, f)=Q_{q}(z, f),
$$

where $U_{q}(z, f), P_{q}(z, f)$, and $Q_{q}(z, f)$ are $q$-difference polynomials such that the total degree $\operatorname{deg} U_{q}(z, f)=n$ in $f(z)$ and its $q$-shifts, whereas $\operatorname{deg} Q_{q}(z, f) \leq n$. Moreover, we assume that $U_{q}(z, f)$ contains just one term of maximal total degree in $f(z)$ and its q-shifts. Then

$$
m\left(r, P_{q}(z, f)\right)=o(T(r, f)),
$$

on a set of logarithmic density 1 .

Lemma 8 (see [10, Theorem 2.2]). Let $f$ be a nonconstant zero-order meromorphic solution of $P_{q}(z, f)=0$, where $P_{q}(z, f)$ is a q-difference polynomial in $f(z)$. If $P_{q}(z, a) \neq \equiv$ for slowly moving target $\alpha(z)$, then

$$
m\left(r, \frac{1}{f-\alpha}\right)=o(T(r, f)),
$$

on a set of logarithmic density 1.
Remark 9 (see [10]). Let $\alpha$ and $f$ be meromorphic functions of zero-order such that $T(r, \alpha)=o(T(r, f))$ on a set of logarithmic density 1 . Then $\alpha$ is called a slowly moving target or a small function with respect to $f$.

Lemma 10 (see [22, Theorems 1.1 and 1.3]). Let $f(z)$ be a nonconstant zero-order meromorphic function and $q \in \mathbb{C} \backslash\{0\}$. Then

$$
\begin{aligned}
& T(r, f(q z))=(1+o(1)) T(r, f(z)), \\
& N(r, f(q z))=(1+o(1)) N(r, f(z)),
\end{aligned}
$$

on a set of lower logarithmic density 1 .

Lemma 11 (Valiron-Mohon'ko, see [33]). Let $f(z)$ be a meromorphic function. Then for all irreducible rational functions in $f$,

$$
R(z, f(z))=\frac{\sum_{i=0}^{m} a_{i}(z) f(z)^{i}}{\sum_{j=0}^{n} b_{j}(z) f(z)^{j}},
$$

with meromorphic coefficients $a_{i}(z), b_{j}(z)$, the characteristic function of $R(z, f(z))$ satisfies

$$
T(r, R(z, f(z)))=d T(r, f)+O(\Psi(r)),
$$

where $d=\max \{m, n\}$ and $\Psi(r)=\max _{i, j}\left\{T\left(r, a_{i}\right), T\left(r, b_{j}\right)\right\}$.

Lemma 12 (see [10, Theorem 1.1]). Let $f(z)$ be a nonconstant zero-order meromorphic function and $q \in \mathbb{C} \backslash\{0\}$. Then

$$
m\left(r, \frac{f(q z)}{f(z)}\right)=S(r, f)
$$

on a set of logarithmic density 1 .

\section{The Proof of Theorem 3}

We first assume that $f(z)$ is of zero-order and a transcendental meromorphic solution of (11).

(i) In view of (11), it follows that

$$
f(z)\left[f(q z)+f\left(\frac{z}{q}\right)\right]=C(z) f(z)+A(z) .
$$

Hence, we conclude from (28) and Lemma 7 that

$$
m\left(r, f(q z)+f\left(\frac{z}{q}\right)\right)=S(r, f)
$$

on a set $F$ of logarithmic density 1 . Since $f(z)$ is of zero-order, then we can deduce by Lemma 10 that

$$
\begin{aligned}
& N\left(r, f(q z)+f\left(\frac{z}{q}\right)\right) \\
& \quad \leq N(r, f(q z))+N\left(r, f\left(\frac{z}{q}\right)\right) \\
& \quad=2(1+o(1)) N(r, f),
\end{aligned}
$$

on a set of lower logarithmic density 1 . 
Since $A(z) \quad \neq \quad 0$ and $A(z), C(z) \in \mathcal{S}(f)$, by applying Lemma 11 for (11), we obtain

$$
T\left(r, f(q z)+f\left(\frac{z}{q}\right)\right)=T(r, f)+S(r, f) .
$$

Thus, it follows from (29) to (31) that

$$
T(r, f) \leq 2(1+o(1)) N(r, f)+S(r, f),
$$

on a set $F$ of logarithmic density 1 . Hence, it means that $f(z)$ has infinitely many poles and

$$
\lambda\left(\frac{1}{f}\right) \geq \sigma(f) .
$$

On the other hand, it yields from (11) that

$$
\begin{aligned}
P_{1}(z, f(z))= & f(z)\left[f(q z)+f\left(\frac{z}{q}\right)\right]-C(z) f(z) \\
& -A(z)=0 .
\end{aligned}
$$

Since $A(z) \not \equiv \quad 0$, then $P_{1}(z, 0)=-A(z) \not \equiv \quad 0$. Thus, by Lemma 8 , it follows that

$$
m\left(r, \frac{1}{f}\right)=S(r, f),
$$

on a set $F$ of logarithmic density 1 . Hence,

$$
N\left(r, \frac{1}{f}\right)=T(r, f)+S(r, f),
$$

on a set $F$ of logarithmic density 1 . Therefore, $f(z)$ has infinitely many zeros and $\lambda(f)=\sigma(f)$.

Next, we will prove that $\lambda\left(1 / \Delta_{q} f\right) \geq \lambda(1 / f)$. Let $z=q w$, then (11) can be rewritten as the form

$$
f\left(q^{2} w\right)+f(w)=\frac{A(q w)}{f(q w)}+C(q w) .
$$

Then it follows from (37) that

$$
\begin{aligned}
& f(q w)\left[f\left(q^{2} w\right)+f(w)\right] \\
& =A(q w)+C(q w) f(q w) .
\end{aligned}
$$

Moreover, it yields $f(q w)=\Delta_{q} f(w)+f(w)$ and $f\left(q^{2} w\right)=$ $\Delta_{q} f(q w)+\Delta_{q} f(w)+f(w)$, where $\Delta_{q} f(w)=f(q w)-f(w)$. Substituting them into (38), we conclude

$$
\begin{aligned}
& {\left[\Delta_{q} f(w)+f(w)\right]\left[\Delta_{q} f(q w)+\Delta_{q} f(w)+2 f(w)\right]} \\
& \quad=A(q w)+C(q w)\left[\Delta_{q} f(w)+f(w)\right],
\end{aligned}
$$

that is,

$$
\begin{aligned}
-2 f(w)^{2} \\
=\left[\Delta_{q} f(q w)+3 \Delta_{q} f(w)-C(q w)\right] f(w) \\
-A(q w) \\
+\left[\Delta_{q} f(q w)+\Delta_{q} f(w)-C(q w)\right] \Delta_{q} f(w) .
\end{aligned}
$$

From the conditions of Theorem 3, and by Lemma 10, we conclude that $f(w)$ is of zero order. Thus, we conclude that $\Delta_{q} f(w), \Delta_{q} f(q w)$ are of zero order and $A(q w), C(q w) \in$ $\delta(f)$, and $\Delta_{q} f(q w)=\Delta_{q}^{2} f(w)+\Delta_{q} f(w)$, where $\Delta_{q}^{2} f(w):=$ $\Delta_{q}\left(\Delta_{q} f(w)\right)$. Since $\Delta_{q} f(w)$ is of zero order, then

$$
N\left(r, \Delta_{q}^{2} f(w)\right) \leq 2 N\left(r, \Delta_{q} f(w)\right)+S(r, f),
$$

on a set $F$ of logarithmic density 1 . Thus, we can deduce from (41) that

$$
N\left(r, \Delta_{q} f(q w)\right) \leq 3 N\left(r, \Delta_{q} f(w)\right)+S(r, f),
$$

on a set $F$ of logarithmic density 1 . Moreover, it follows from (40) and (42) that

$$
\begin{aligned}
2 N & (r, f(w)) \\
& =N\left(r,\left[\Delta_{q} f(q w)+3 \Delta_{q} f(w)-C(q w)\right] f(w)\right. \\
& -A(q w) \\
& \left.+\left[\Delta_{q} f(q w)+\Delta_{q} f(w)-C(q w)\right] \Delta_{q} f(w)\right) \\
& \leq N(r, f(w))+9 N\left(r, \Delta_{q} f(w)\right)+S(r, f),
\end{aligned}
$$

on a set $F$ of logarithmic density 1; that is,

$$
N(r, f(w)) \leq 9 N\left(r, \Delta_{q} f(w)\right)+S(r, f),
$$

on a set $F$ of logarithmic density 1 . Then, it means that $\Delta_{q} f(w)$ has infinitely many poles and

$$
\lambda\left(\frac{1}{\Delta_{q} f(w)}\right) \geq \lambda\left(\frac{1}{f(w)}\right) .
$$

Since $f(w)$ is of zero order, then we conclude from Lemma 10 that

$$
\begin{aligned}
\lambda\left(\frac{1}{\Delta_{q} f(w)}\right) & =\lambda\left(\frac{1}{\Delta_{q} f(z / q)}\right)=\lambda\left(\frac{1}{\Delta_{q} f(z)}\right), \\
\lambda\left(\frac{1}{f(w)}\right) & =\lambda\left(\frac{1}{f(z / q)}\right)=\lambda\left(\frac{1}{f(z)}\right) .
\end{aligned}
$$

Hence, it yields

$$
\lambda\left(\frac{1}{\Delta_{q} f}\right) \geq \lambda\left(\frac{1}{f}\right) .
$$

Thus, by (33) and (47) we conclude that

$$
\lambda\left(\frac{1}{\Delta_{q} f}\right) \geq \lambda\left(\frac{1}{f}\right) \geq \sigma(f) .
$$

$T\left(r, \Delta_{q} f\right) \leq 2 T(r, f)+S(r, f)$ on a set $F$ of logarithmic density 1: that is, $\sigma(f) \geq \sigma\left(\Delta_{q} f\right)$. Thus, combining this and (48), we conclude that $\Delta_{q} f(z)$ has infinitely many poles and

$$
\lambda(f)=\lambda\left(\frac{1}{f}\right)=\lambda\left(\frac{1}{\Delta_{q} f}\right) .
$$


Next, we prove that

$$
\lambda\left(\frac{1}{\Delta_{q}^{2} f}\right)=\lambda\left(\frac{1}{\Delta_{q} f / f}\right)=\lambda\left(\frac{1}{\Delta_{q}^{2} f / f}\right) .
$$

At first, it can be seen that $A(z)+C(z) f(z)$ and $f(z)^{2}$ are mutually prime polynomials in $f(z)$, where $A(z), C(z) \in$ $\mathcal{S}(f)$. In fact, taking $u(z, f)=A(z)-C(z) f(z)$ and $v(z, f)=$ $C(z)^{2}$, it yields $u(z, f)(A(z)+C(z) f(z))+v(z, f) f(z)^{2}=$ $A(z)^{2}$. Thus, in view of (11) and Lemma 11, we deduce

$$
\begin{aligned}
2 T(r, f) & =T\left(r, \frac{A(z)+C(z) f(z)}{f(z)^{2}}\right)+S(r, f) \\
& =T\left(r, \frac{f(q z)+f(z / q)}{f(z)}\right)+S(r, f) \\
& \leq 2 T\left(r, \frac{f(q z)}{f(z)}\right)+S(r, f) \\
& =2 T\left(r, \frac{\Delta_{q} f}{f}\right)+S(r, f),
\end{aligned}
$$

that is,

$$
T(r, f) \leq T\left(r, \frac{\Delta_{q} f}{f}\right)+S(r, f) .
$$

Hence, by Lemma 12, it yields

$$
\begin{aligned}
N\left(r, \frac{\Delta_{q} f}{f}\right) & =T\left(r, \frac{\Delta_{q} f}{f}\right)-m\left(r, \frac{\Delta_{q} f}{f}\right) \\
& \geq T(r, f)+S(r, f), \\
N\left(r, \frac{\Delta_{q} f}{f}\right) & \leq T\left(r, \frac{\Delta_{q} f}{f}\right)+S(r, f) \\
& \leq 3 T(r, f)+S(r, f) .
\end{aligned}
$$

Besides, in view of (11), it follows that

$$
\begin{aligned}
\Delta_{q}^{2} f\left(\frac{z}{q}\right) & =\Delta_{q} f(z)-\Delta_{q} f\left(\frac{z}{q}\right) \\
& =\frac{-2 f(z)^{2}+C(z) f(z)+A(z)}{f(z)},
\end{aligned}
$$

and by Lemmas 10 and 11, it yields

$$
\begin{aligned}
2 T(r, f(z))= & T\left(r, \frac{-2 f(z)^{2}+C(z) f(z)+A(z)}{f(z)}\right) \\
& +S(r, f) \\
= & T\left(r, \Delta^{2} f\left(\frac{z}{q}\right)\right)+S(r, f) \\
= & T\left(r, \Delta_{q}^{2} f(z)\right)+S(r, f) .
\end{aligned}
$$

Thus, it follows that

$$
\begin{aligned}
3 T(r, f)+S(r, f) & \geq N\left(r, \Delta_{q}^{2} f\right) \\
& =T\left(r, \Delta_{\mathrm{q}}^{2} f\right)-m\left(r, \Delta_{q}^{2} f\right) \\
& \geq 2 T(r, f)-T(r, f)+S(r, f) \\
& =T(r, f)+S(r, f) .
\end{aligned}
$$

Finally, similar to the above argument, we have

$$
\begin{aligned}
2 T(r, f)+S(r, f) & \geq N\left(r, \frac{\Delta_{q}^{2} f}{f}\right) \\
& =T\left(r, \frac{\Delta_{q}^{2} f}{f}\right)-m\left(r, \frac{\Delta_{q}^{2} f}{f}\right) \\
& \geq T\left(r, \Delta_{q}^{2} f\right)-T(r, f)+S(r, f) \\
& =T(r, f)+S(r, f) .
\end{aligned}
$$

Thus, we can conclude from (53), (56), and (57) that each of $\Delta_{q}^{2} f, \Delta_{q} f / f, \Delta_{q}^{2} f / f$ has infinitely many poles and

$$
\lambda\left(\frac{1}{\Delta_{q}^{2} f}\right)=\lambda\left(\frac{1}{\Delta_{q} f / f}\right)=\lambda\left(\frac{1}{\Delta_{q}^{2} f / f}\right) .
$$

(ii) Set $g(z)=f(z)-z$. Then $g(z)$ is a zero-order transcendental meromorphic function with $\sigma(g)=\sigma(f)$ and $\tau(f)=\lambda(g)$. Substituting $f(z)=g(z)+z$ into (11), we have

$$
\begin{aligned}
P_{2}(z, g(z))= & (g(z)+z)\left[g(q z)+g\left(\frac{z}{q}\right)\right] \\
& +\left[\left(q+\frac{1}{q}\right) z-C(z)\right] g(z) \\
& +\left(q+\frac{1}{q}\right) z^{2}-z C(z)-A(z)=0 .
\end{aligned}
$$

Since $P_{2}(z, 0)=(q+1 / q) z^{2}-z C(z)-A(z) \not \equiv 0$, then by Lemma 8 , it yields

$$
m\left(r, \frac{1}{g}\right)=S(r, f)
$$

on a set $F$ of logarithmic density 1. By using the same argument as in the proof of Theorem 3(i), we conclude

$$
N\left(r, \frac{1}{g}\right)=T(r, f)+S(r, f)
$$

on a set $F$ of logarithmic density 1, which implies $f$ has infinitely many fixed points and $\tau(f)=\lambda(g)=\sigma(f)$.

Therefore, this completes the proof of Theorem 3.

\section{The Proof of Theorem 4}

We assume that $f(z)$ is a zero-order transcendental meromorphic solution of (14). 
(i) In view of (14), it follows that

$$
\begin{aligned}
P_{q}(z, f):= & f(z)^{2}\left[f(q z)+f\left(\frac{z}{q}\right)\right]+A(z) f(z) \\
& +C(z)-f(q z)-f\left(\frac{z}{q}\right)=0 .
\end{aligned}
$$

Since $C(z) \not \equiv 0$, then it follows that

$$
P_{q}(z, 0) \equiv C(z) \not \equiv 0 .
$$

Thus, we can conclude by Lemma 8 that

$$
m\left(r, \frac{1}{f}\right)=S(r, f)
$$

on a set $F$ of logarithmic density 1 . Hence,

$$
N\left(r, \frac{1}{f}\right)=T(r, f)+S(r, f)
$$

on a set $F$ of logarithmic density 1 . Thus, it means that $f(z)$ has infinitely many zeros and $\lambda(f)=\sigma(f)$.

On the other hand, it follows from (14) that

$$
\begin{aligned}
& f(z)^{2}\left[f(q z)+f\left(\frac{z}{q}\right)\right] \\
& \quad=f(q z)+f\left(\frac{z}{q}\right)-A(z) f(z)-C(z) .
\end{aligned}
$$

Then in view of (66) and Lemma 7, we have

$$
m\left(r, f(q z)+f\left(\frac{z}{q}\right)\right)=S(r, f) .
$$

And by applying Lemma 11 for (14), it yields

$$
T\left(r, f(q z)+f\left(\frac{z}{q}\right)\right)=2 T(r, f)+S(r, f) .
$$

Then, we conclude by Lemma 10 that

$$
\begin{aligned}
& N\left(r, f(q z)+f\left(\frac{z}{q}\right)\right) \\
& \quad \leq N(r, f(q z))+N\left(r, f\left(\frac{z}{q}\right)\right) \\
& \quad=2(1+o(1)) N(r, f)
\end{aligned}
$$

on a set of lower logarithmic density 1 . Thus, combining (67) and (68), it follows that

$$
T(r, f) \leq 2(1+o(1)) N(r, f)+S(r, f) .
$$

Hence, $f(z)$ has infinitely many poles and

$$
\sigma(f) \leq \lambda\left(\frac{1}{f}\right)
$$

Next, we will prove that $\lambda\left(1 / \Delta_{q} f\right) \geq \lambda(1 / f)$. Let $z=q w$; then (14) can be rewritten as the following form:

$$
f\left(q^{2} w\right)+f(w)=\frac{A(q w) f(q w)+C(q w)}{1-f(q w)^{2}} .
$$

Then from (72) it follows that

$$
\begin{aligned}
& f(q w)^{2}\left[f\left(q^{2} w\right)+f(w)\right] \\
& \quad=f\left(q^{2} w\right)+f(w)-A(q w) f(q w)-C(q w),
\end{aligned}
$$

and $f(q w)=\Delta_{q} f(w)+f(w)$ and $f\left(q^{2} w\right)=\Delta_{q} f(q w)+$ $\Delta_{q} f(w)+f(w)$. Substituting them into (73), we have

$$
\begin{aligned}
{\left[\Delta_{q} f(w)+f(w)\right]^{2}\left[\Delta_{q} f(q w)+\Delta_{q} f(w)+2 f(w)\right] } \\
=\Delta_{q} f(q w)+\Delta_{q} f(w)+2 f(w) \\
\quad-A(q w)\left[\Delta_{q} f(w)+f(w)\right]-C(q w) .
\end{aligned}
$$

Thus, it follows that

$$
-2 f(w)^{3}=P(w) f(w)+Q(w) \Delta_{q} f(w)+R(w),
$$

where

$$
\begin{aligned}
P(w)= & {\left[\Delta_{q} f(q w)+5 \Delta_{q} f(w)\right] f(w) } \\
& +4\left(\Delta_{q} f(w)\right)^{2}+2 \Delta_{q} f(w) \Delta_{q} f(q w) \\
& +A(q w)-2, \\
Q(w)= & \Delta_{q} f(q w) \Delta_{q} f(w)+\left(\Delta_{q} f(w)\right)^{2}+A(q w) \\
& -1, \\
R(w)= & C(q w)-\Delta_{q} f(q w) .
\end{aligned}
$$

Hence, it follows by Lemma 10 and from (75) that

$$
\begin{aligned}
3 N & (r, f(w)) \\
= & N\left(r, P(w) f(w)+Q(w) \Delta_{q} f(w)+R(w)\right) \\
\leq & 2 N(r, f(w))+19 N\left(r, \Delta_{q} f(w)\right)+O(\log r) \\
& +S(r, f),
\end{aligned}
$$

that is,

$$
N(r, f(w)) \leq 19 N\left(r, \Delta_{q} f(w)\right)+S(r, f) .
$$

Therefore, it follows from (78) that $\Delta_{q} f$ has infinitely many poles and

$$
\lambda\left(\frac{1}{\Delta_{q} f}\right) \geq \lambda\left(\frac{1}{f}\right)
$$


Since $f(w)$ is of zero-order, by using the same argument as in the proof of Theorem 3, we conclude that $\Delta_{q} f(z)$ has infinitely many poles and

$$
\lambda\left(\frac{1}{f}\right)=\lambda\left(\frac{1}{\Delta_{q} f}\right)=\sigma\left(\Delta_{q} f\right)=\sigma(f) .
$$

Here, we will prove that

$$
\lambda\left(\frac{1}{\Delta_{q}^{2} f}\right)=\lambda\left(\frac{1}{\Delta_{q} f / f}\right)=\lambda\left(\frac{1}{\Delta_{q}^{2} f / f}\right) .
$$

Similarly, let $A(z), C(z) \in \mathcal{S}(f), u(z, f)=A(z)^{2} f(z)^{2}-$ $A(z) C(z) f(z)+C(z)^{2}-A(z)^{2}$, and $v(z, f)=A(z)^{3}$; then $u(z, f)(A(z) f(z)+C(z))+v(z, f) f(z)\left(1-f(z)^{2}\right)=$ $C(z)\left(C(z)^{2}-A(z)^{2}\right)$. Hence, it can be seen that $A(z) f(z)+$ $C(z)$ and $f(z)\left(1-f(z)^{2}\right)$ are mutually prime polynomials in $f(z)$. Thus, from (14) and by Lemma 11, it follows that

$$
\begin{aligned}
3 T(r, f) & =T\left(r, \frac{A(z)+C(z) f(z)}{f(z)\left(1-f(z)^{2}\right)}\right)+S(r, f) \\
& =T\left(r, \frac{f(q z)+f(z / q)}{f(z)}\right)+S(r, f) \\
& \leq 2 T\left(r, \frac{f(q z)}{f(z)}\right)+S(r, f) \\
& =2 T\left(r, \frac{\Delta_{q} f}{f}\right)+S(r, f),
\end{aligned}
$$

that is,

$$
\frac{3}{2} T(r, f) \leq T\left(r, \frac{\Delta_{q} f}{f}\right)+S(r, f) .
$$

Hence, in view of Lemmas 8 and 12, it yields

$$
\begin{aligned}
N\left(r, \frac{\Delta_{q} f(z)}{f(z)}\right)= & T\left(r, \frac{\Delta_{q} f(z)}{f(z)}\right) \\
& -m\left(r, \frac{\Delta_{q} f(z)}{f(z)}\right) \\
& \geq \frac{3}{2} T(r, f(z))+S(r, f), \\
N\left(r, \frac{\Delta_{q} f}{f}\right) & \leq T\left(r, \frac{\Delta_{q} f}{f}\right)+S(r, f) \\
& \leq 3 T(r, f)+S(r, f) .
\end{aligned}
$$

Besides, in view of (14), we have

$$
\begin{aligned}
\Delta_{q}^{2} f\left(\frac{z}{q}\right) & =\Delta_{q} f(z)-\Delta_{q} f\left(\frac{z}{q}\right) \\
& =\frac{2 f(z)^{3}+(A(z)-2) f(z)+C(z)}{1-f(z)^{2}},
\end{aligned}
$$

and then by Lemma 11, it follows that

$$
\begin{aligned}
3 T(r, f(z)) & \\
= & T\left(r, \frac{2 f(z)^{3}+(A(z)-2) f(z)+C(z)}{1-f(z)^{2}}\right) \\
& +S(r, f)=T\left(r, \Delta_{q}^{2} f\left(\frac{z}{q}\right)\right)+S(r, f) \\
= & T\left(r, \Delta_{q}^{2} f(z)\right)+S(r, f) .
\end{aligned}
$$

Thus, by Lemma 10 and the above equalities, it yields

$$
\begin{aligned}
3 T(r, f)+S(r, f) & \geq N\left(r, \Delta_{q}^{2} f\right) \\
& =T\left(r, \Delta_{q}^{2} f(z)\right)-m\left(r, \Delta_{q}^{2} f\right) \\
& \geq 3 T(r, f)-T(r, f)+S(r, f) \\
& =2 T(r, f)+S(r, f) .
\end{aligned}
$$

Finally, similar to the above argument, it follows that

$$
\begin{aligned}
3 T(r, f)+S(r, f) & \geq N\left(r, \frac{\Delta_{q}^{2} f}{f}\right) \\
& =T\left(r, \frac{\Delta_{q}^{2} f}{f}\right)-m\left(r, \frac{\Delta_{q}^{2} f}{f}\right) \\
& \geq T\left(r, \Delta_{q}^{2} f\right)-T(r, f)+S(r, f) \\
& =2 T(r, f)+S(r, f) .
\end{aligned}
$$

Thus, in view of (84), (87), and (88), we can deduce that each of $\Delta_{q}^{2} f, \Delta_{q} f / f, \Delta_{q}^{2} f / f$ has infinitely many poles and

$$
\lambda\left(\frac{1}{\Delta_{q}^{2} f}\right)=\lambda\left(\frac{1}{\Delta_{q} f / f}\right)=\lambda\left(\frac{1}{\Delta_{q}^{2} f / f}\right) .
$$

(ii) Set $g(z)=f(z)-z$. Thus, $g(z)$ is a zero-order transcendental meromorphic function with $\sigma(g)=\sigma(f)$ and $\tau(f)=\lambda(g)$. Substituting $f(z)=g(z)+z$ into (14), it follows that

$$
\begin{aligned}
P_{2}(z, g(z))= & (g(z)+z)^{2}\left[g(q z)+g\left(\frac{z}{q}\right)\right] \\
& +g(z)[g(z)+2 z]\left(q+\frac{1}{q}\right) z \\
& -\left[g(q z)+g\left(\frac{z}{q}\right)\right]+A(z) g(z) \\
& +\left(q+\frac{1}{q}\right)\left(z^{3}-z\right)+z A(z)+C(z) .
\end{aligned}
$$

Since $P_{2}(z, 0)=(q+1 / q)\left(z^{3}-z\right)+z A(z)+C(z) \not \equiv 0$, then in view of Lemma 8 , it follows that

$$
m\left(r, \frac{1}{g}\right)=S(r, f)
$$


on a set $F$ of logarithmic density 1 . By using the same argument as in the proof of Theorem 3(i), it follows that

$$
N\left(r, \frac{1}{g}\right)=T(r, f)+S(r, f)
$$

on a set $F$ of logarithmic density 1 , which implies that $f(z)$ has infinitely many fixed points and $\tau(f)=\lambda(g)=\sigma(f)$.

Therefore, this completes the proof of Theorem 4 .

\section{The Proof of Theorem 5}

We first assume that $f(z)$ is a zero-order transcendental meromorphic solution of (17).

(i) In view of (17), it follows that

$$
f(z)^{2}\left[f(q z)+f\left(\frac{z}{q}\right)\right]=A(z) f(z)+C(z) .
$$

Thus, we conclude from (93) and Lemma 7 that

$$
m\left(r, f(q z)+f\left(\frac{z}{q}\right)\right)=S(r, f) .
$$

And by Lemma 10 it follows that

$$
\begin{aligned}
& N\left(r, f(q z)+f\left(\frac{z}{q}\right)\right) \\
& \quad \leq N(r, f(q z))+N\left(r, f\left(\frac{z}{q}\right)\right) \\
& \quad=2(1+o(1)) N(r, f),
\end{aligned}
$$

on a set of lower logarithmic density 1 . that

If $C(z) \neq 0$, then by applying Lemma 11 for (17), it follows

$$
T\left(r, f(q z)+f\left(\frac{z}{q}\right)\right)=2 T(r, f)+S(r, f) .
$$

Hence, from (94) to (96), it yields

$$
T(r, f) \leq(1+o(1)) N(r, f)+S(r, f) .
$$

If $A(z) \neq 0$ and $C(z) \equiv 0$, by using the same argument as in the proof of Theorem 3(i), it follows that

$$
T(r, f) \leq 2(1+o(1)) N(r, f)+S(r, f) .
$$

Therefore, from (97) and (98), it means that $f(z)$ has infinitely many poles and

$$
\sigma(f) \leq \lambda\left(\frac{1}{f}\right)
$$

Now, we will prove that $\lambda\left(1 / \Delta_{q} f\right) \geq \lambda(1 / f)$ as follows.

Set $z=q w$, by using the same argument as in Theorem 3(i), it yields

$$
-2 f(w)^{3}=P(w) f(w)+Q(w) \Delta_{q} f(w)+R(w),
$$

where

$$
\begin{aligned}
P(w)= & {\left[\Delta_{q} f(q w)+5 \Delta_{q} f(w)\right] f(w) } \\
& +4\left(\Delta_{q} f(w)\right)^{2}+2 \Delta_{q} f(w) \Delta_{q} f(q w) \\
& -A(q w), \\
Q(w)= & {\left[\Delta_{q} f(q w)+\Delta_{q} f(w)\right] \Delta_{q} f(w)-A(q w), } \\
R(w)= & -C(q w) .
\end{aligned}
$$

Hence, we conclude by Lemma 10 and from (100) that

$$
\begin{aligned}
3 N & (r, f(w)) \\
= & N\left(r, P(w) f(w)+Q(w) \Delta_{q} f(w)+R(w)\right) \\
\leq & 2 N(r, f(w))+15 N\left(r, \Delta_{q} f(w)\right)+O(\log r) \\
& +S(r, f),
\end{aligned}
$$

that is,

$$
N(r, f(w)) \leq 15 N\left(r, \Delta_{q} f(w)\right)+S(r, f) .
$$

Thus, it follows from (103) that

$$
\lambda\left(\frac{1}{\Delta_{q} f(w)}\right) \geq \lambda\left(\frac{1}{f(w)}\right) .
$$

By using the same argument as in the proof of Theorem 3(i), we can conclude that $\Delta_{q} f$ has infinitely many poles, and

$$
\begin{aligned}
\lambda\left(\frac{1}{f}\right) & =\lambda\left(\frac{1}{\Delta_{q} f}\right)=\sigma\left(\Delta_{q} f\right)=\sigma(f), \\
\lambda\left(\frac{1}{\Delta_{q}^{2} f}\right) & =\lambda\left(\frac{1}{\Delta_{q} f / f}\right)=\lambda\left(\frac{1}{\Delta_{q}^{2} f / f}\right) .
\end{aligned}
$$

(ii) Let $g(z)=f(z)-z$. Thus, $g(z)$ is a zero-order transcendental meromorphic function with $\sigma(g)=\sigma(f)$ and $\tau(f)=\lambda(g)$. Substituting $f(z)=g(z)+z$ into (17), it follows that

$$
\begin{aligned}
P_{2}(z, g(z))= & (g(z)+z)^{2}\left[g(q z)+g\left(\frac{z}{q}\right)\right] \\
& +g(z)[g(z)+2 z]\left(q+\frac{1}{q}\right) z \\
& -A(z) g(q z)+\left(q+\frac{1}{q}\right) z^{3} \\
& -z A(z)-C(z) .
\end{aligned}
$$

Since $P_{2}(z, 0)=(q+1 / q) z^{3}-z A(z)-C(z) \not \equiv 0$, then it yields by Lemma 8 that

$$
m\left(r, \frac{1}{g}\right)=S(r, f)
$$


on a set $F$ of logarithmic density 1 . By using the same argument as in the proof of Theorem 3(i), we have

$$
N\left(r, \frac{1}{g}\right)=T(r, f)+S(r, f)
$$

on a set $F$ of logarithmic density 1 , which implies that $f(z)$ has infinitely many fixed points and $\tau(f)=\lambda(g)=\sigma(f)$.

Therefore, this completes the proof of Theorem 5 .

\section{Conflicts of Interest}

The authors declare that they have no conflicts of interest in the manuscript.

\section{Authors' Contributions}

Hong Yan Xu and Peng Jun Zhao completed the main part of this article, and Hong Yan Xu and Peng Jun Zhao corrected the main theorems. All authors read and approved the final manuscript.

\section{Acknowledgments}

This work was supported by the National Natural Science Foundation of China (11561033), the Natural Science Foundation of Jiangxi Province in China (20151BAB201008), and the Foundation of Education Department of Jiangxi (GJJ160914, GJJ170759, GJJ161603, and GJJ170788) of China.

\section{References}

[1] P. Painlevé, "Mémoire sur les équations différentielles dont lintégrale générale est uniforme," Bull. Soc. Math. France, vol. 28, no. 1900, pp. 201-261, 1900.

[2] A. S. Fokas, "From continuous to discrete Painlevé equations," Journal of Mathematical Analysis and Applications, vol. 180, pp. 342-360, 1993.

[3] B. Grammaticos, F. W. Nijhoff, and A. Ramani, "Discrete Painlevé equations, The Painlevé property," in CRM Series in Mathematical Physics, pp. 413-516, Springer, New York, NY, USA, 1999.

[4] Y. M. Chiang and S. J. Feng, "On the Nevanlinna characteristic of $f(z+\eta)$ and difference equations in the complex plane," Ramanujan Journal, vol. 16, pp. 105-129, 2008.

[5] R. G. Halburd and R. J. Korhonen, "Finite order solutions and the discrete Painlevé equations," Proceedings of the London Mathematical Society, vol. 94, pp. 443-474, 2007.

[6] J. Heittokangas, R. Korhonen, I. Laine, J. Rieppo, and K. Tohge, "Complex difference equations of Malmquist type," Computational Methods and Function Theory, vol. 1, no. 1, pp. 27-39, 2001.

[7] I. Laine and C. C. Yang, "Value distribution of difference polynomials," Proceedings of the Japan Academy, Series A, vol. 83, pp. 148-151, 2007.

[8] R. G. Halburd and R. J. Korhonen, "Difference analogue of the lemma on the logarithmic derivative with applications to difference equations," Journal of Mathematical Analysis and Applications, vol. 314, no. 2, pp. 477-487, 2006.
[9] R. G. Halburd and R. J. Korhonen, "Nevanlinna theory for the difference operator," Annales Academice Scientiarum Fennicee Mathematica, vol. 31, no. 2, pp. 463-478, 2006.

[10] D. C. Barnett, R. G. Halburd, R. J. Korhonen, and W. Morgan, "Nevanlinna theory for the $q$-difference operator and meromorphic solutions of $q$-difference equations," Proc. Roy. Soc. Edin. Sect. A Math, vol. 137, pp. 457-474, 2007.

[11] L. Y. Gao and M. L. Wang, "The transcendental meromorphic solutions of composite functional equations," Journal of Jiangxi Normal University. Natural Sciences, vol. 40, no. 6, pp. 587-590, 2016.

[12] G. G. Gundersen, J. Heittokangas, I. Laine, J. Rieppo, and D. Q. Yang, "Meromorphic solutions of generalized Schröder equations," Aequationes Mathematicae, vol. 63, pp. 110-135, 2002.

[13] I. Laine and C. C. Yang, "Clunie theorems for difference and $q$ difference polynomials," J. London Math. Soc, vol. 76, no. 2, pp. 556-566, 2007.

[14] L. W. Liao and C. C. Yang, "Some new and old (unsolved) problems and conjectures on factorization theory, dynamics and functional equations of meromorphic functions," Journal of Jiangxi Normal University. Natural Sciences, vol. 41, no. 3, pp. 242-247, 2017

[15] K. Liu and T. Cao, "Values sharing results on $q$-difference and derivative of meromorphic functions," Hacettepe Journal of Mathematics and Statistics, vol. 46, no. 6, pp. 1719-1728, 2016.

[16] K. Liu and X.-G. Qi, "Meromorphic solutions of $q$-shift difference equations," Annales Polonici Mathematici, vol. 101, no. 3, pp. 215-225, 2011.

[17] K. Liu, "Entire solutions of Fermat type q-difference differential equations," Electronic Journal of Differential Equations, vol. 2013, no. 59, pp. 1-10, 2013.

[18] L. Q. Luo and X. M. Zheng, "Value distribution of meromorphic solutions of homogeneous and non-homogeneous complex linear differential-difference equations," Open Math, vol. 14, pp. 970-976, 2016.

[19] X. Qi, K. Liu, and L. Yang, "Value sharing results of a meromorphic function $f(z)$ and $f(q z)$," Bulletin of the Korean Mathematical Society, vol. 48, no. 6, pp. 1235-1243, 2011.

[20] M. Ru, "The recent progress in Nevanlinna theory," Journal of Jiangxi Normal University. Natural Sciences, vol. 42, no. 1, pp. 111, 2018.

[21] J. Wang, K. Xia, and F. Long, "The poles of meromorphic solutions of Fermat type differential-difference equations," Journal of Jiangxi Normal University. Natural Sciences, vol. 40, no. 5, pp. 497-499, 2016.

[22] J. Zhang and R. Korhonen, "On the Nevanlinna characteristic of $f(q z)$ and its applications," Journal of Mathematical Analysis and Applications, vol. 369, no. 2, pp. 537-544, 2010.

[23] X. M. Zheng and Z. X. Chen, "Some properties of meromorphic solutions of $q$-difference equations," Journal of Mathematical Analysis and Applications, vol. 361, pp. 472-480, 2010.

[24] X. Zheng and Z. Chen, "On properties of $q$-difference equations," Acta Mathematica Scientia, vol. 32, no. 2, pp. 724-734, 2012.

[25] X.-M. Zheng and H.-Y. Xu, "Results on the deficiencies of some differential-difference polynomials of meromorphic functions," Open Mathematics, vol. 14, pp. 100-108, 2016.

[26] X. M. Zheng, H. Y. Xu, and H. Wang, "On properties of meromorphic solutions for a certain q-difference Painlevé equation," Journal of Computational Analysis and Applications, vol. 23, no. 1, pp. 42-51, 2017. 
[27] W. K. Hayman, Meromorphic Functions, Clarendon Press, Oxford, UK, 1964.

[28] L. Yang, Value Distribution Theory, Springer-Verlag, Berlin, Germany, 1993.

[29] H. X. Yi and C. C. Yang, Uniqueness Theory of Meromorphic Functions, Kluwer Academic Publishers, Dordrecht, The Netherlands, 2003, Science Press, Beijing, China, 1995.

[30] Z. X. Chen and K. H. Shon, "Value distribution of meromorphic solutions of certain difference Painlevé equations," Journal of Mathematical Analysis and Applications, vol. 364, pp. 556-566, 2010.

[31] Q. Li and Z. B. Huang, "Some results on a certain type of difference equation originated from difference Painlevé I equation," Advances in Difference Equations, vol. 2015, p. 276, 2015.

[32] X. G. Qi and L. Z. Yang, "Properties of meromorphic solutions of q-difference equations," Electronic Journal of Differential Equations, vol. 2015, no. 59, pp. 1-9, 2015.

[33] I. Laine, Nevanlinna Theory and Complex Differential Equations, Walter De Gruyter, Berlin, Germany, 1993. 


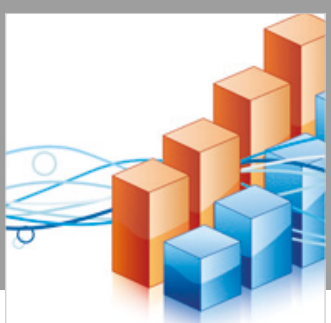

Advances in

Operations Research

\section{-n-m}
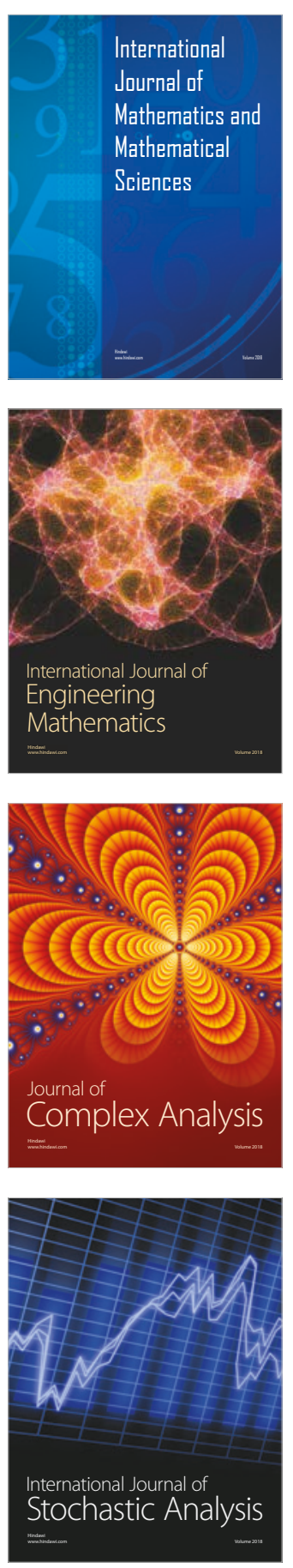
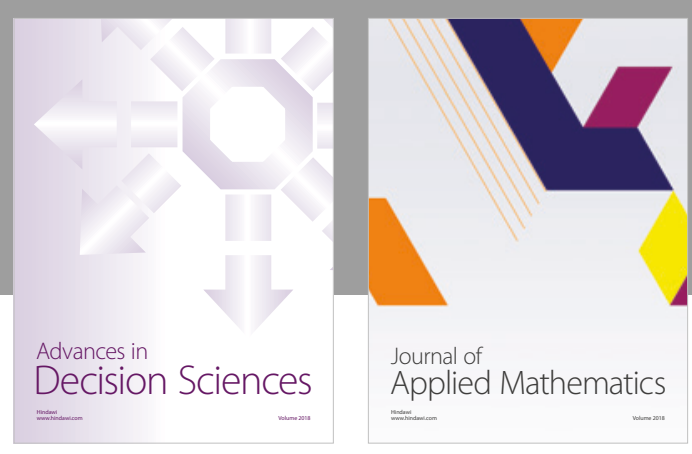

Journal of

Applied Mathematics
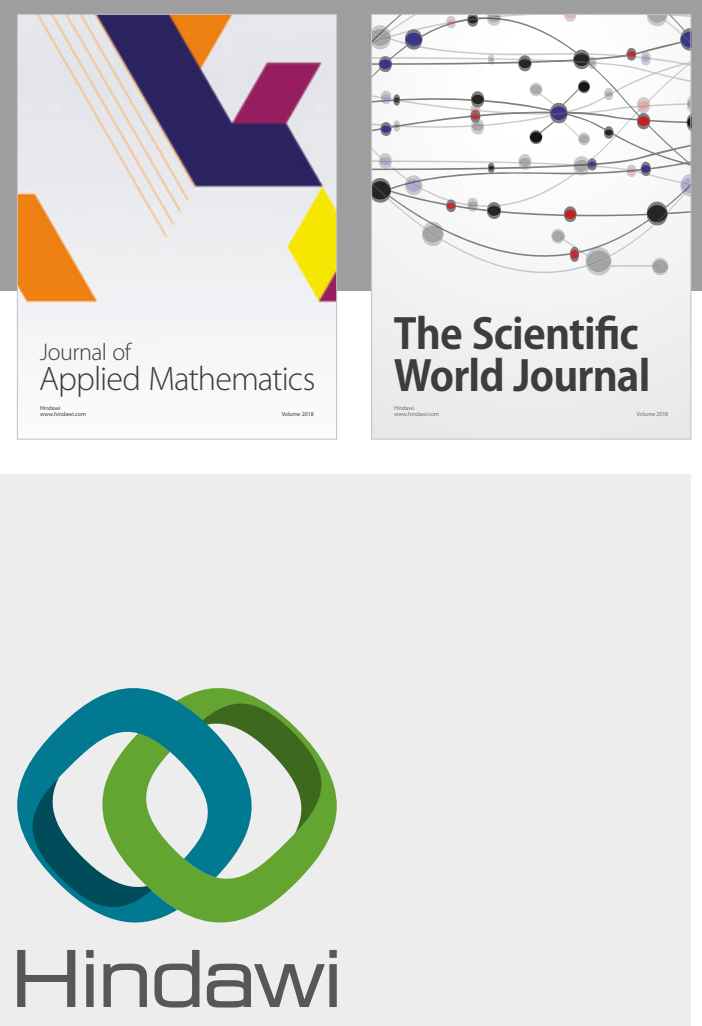

Submit your manuscripts at

www.hindawi.com

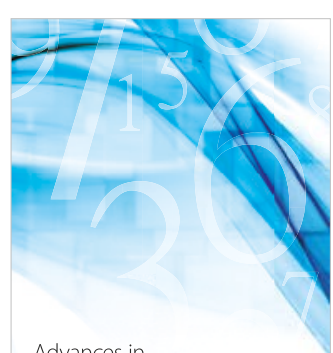

Advances in
Numerical Analysis
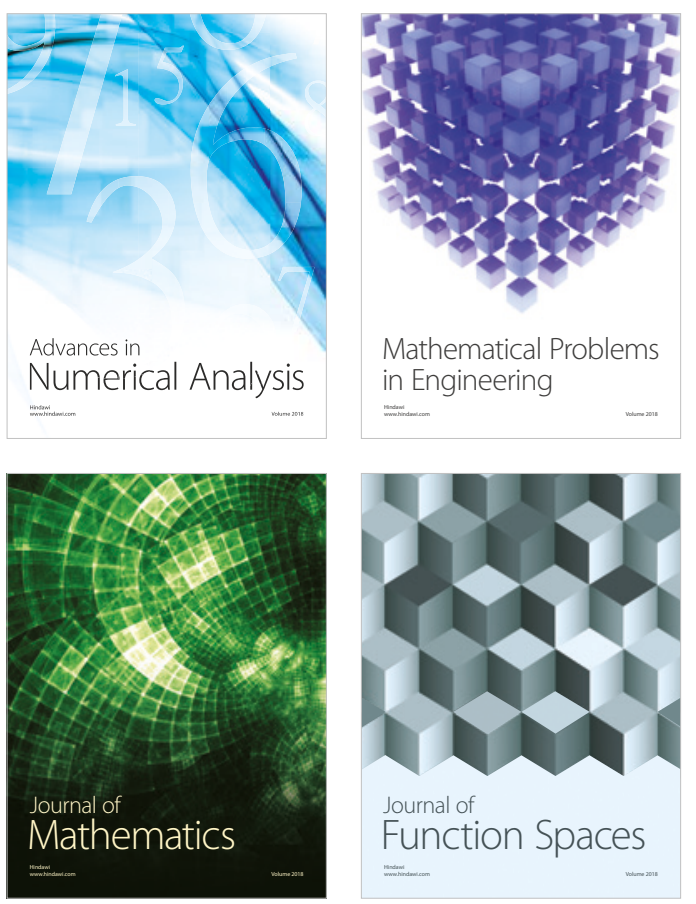

Mathematical Problems in Engineering

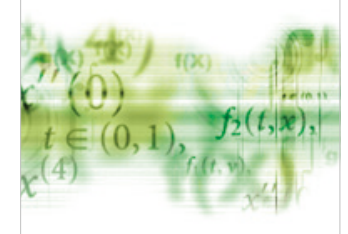

International Journal of

Differential Equations

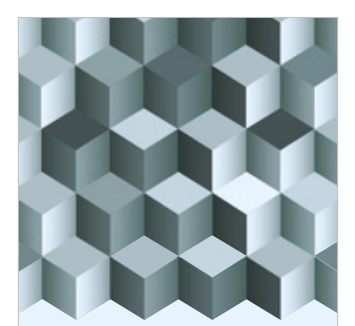

Journal of

Function Spaces



The Scientific

World Journal

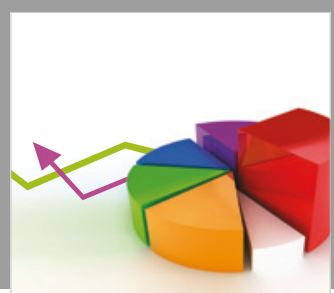

Journal of

Probability and Statistics
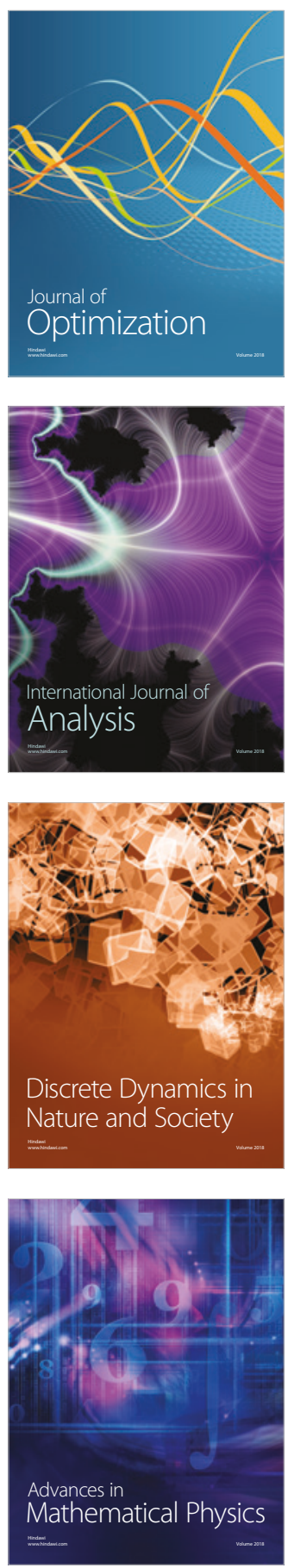